\title{
Computer-Aided Quantification of Interstitial Lung Disease from High Resolution Computed Tomography Images in Systemic Sclerosis: Correlation with Visual Reader-Based Score and Physiologic Tests
}

\author{
Fausto Salaffi, ${ }^{1}$ Marina Carotti, ${ }^{2}$ Silvia Bosello, ${ }^{3}$ Alessandro Ciapetti, ${ }^{1}$ Marwin Gutierrez, \\ Elisabetta Bichisecchi, ${ }^{2}$ Gianmarco Giuseppetti, ${ }^{2}$ and Gianfranco Ferraccioli ${ }^{3}$
}

${ }^{1}$ Department of Rheumatology, Polytechnic University of the Marche, Ancona, Italy

${ }^{2}$ Department of Radiology, Polytechnic University of the Marche, Ancona, Italy

${ }^{3}$ Division of Rheumatology, School of Medicine, Catholic University of the Sacred Heart, Rome, Italy

Correspondence should be addressed to Fausto Salaffi; fausto.salaffi@gmail.com

Received 30 July 2014; Revised 29 October 2014; Accepted 31 October 2014

Academic Editor: Hiroshi Tanaka

Copyright (C) 2015 Fausto Salaffi et al. This is an open access article distributed under the Creative Commons Attribution License, which permits unrestricted use, distribution, and reproduction in any medium, provided the original work is properly cited.

Objective. To evaluate the performance of a computerized-aided method (CaM) for quantification of interstitial lung disease (ILD) in patients with systemic sclerosis and to determine its correlation with the conventional visual reader-based score (CoVR) and the pulmonary function tests (PFTs). Methods. Seventy-nine patients were enrolled. All patients underwent chest high resolution computed tomography (HRCT) scored by two radiologists adopting the CoVR. All HRCT images were then analysed by a CaM using a DICOM software. The relationships among the lung segmentation analysis, the readers, and the PFTs results were calculated using linear regression analysis and Pearson's correlation. Receiver operating curve analysis was performed for determination of CaM extent threshold. Results. A strong correlation between CaM and CoVR was observed $(P<0.0001)$. The CaM showed a significant negative correlation with forced vital capacity $(\mathrm{FVC})(P<0.0001)$ and the single breath carbon monoxide diffusing capacity of the lung (DLco) $(P<0.0001)$. A CaM optimal extent threshold of $20 \%$ represented the best compromise between sensitivity (75.6\%) and specificity (97.4\%). Conclusions. CaM quantification of SSc-ILD can be useful in the assessment of extent of lung disease and may provide reliable tool in daily clinical practice and clinical trials.

\section{Introduction}

Systemic sclerosis (SSc) is a heterogeneous autoimmune disorder of unknown aetiology that is characterized by musculoskeletal involvement, vascular dysfunction, cutaneous and visceral fibrosis [1]. Interstitial lung disease (ILD) was reported in up to $70 \%$ of patients with SSc and frequently it can be cause of death of these patients [1-3].

High resolution computed tomography (HRCT) is currently the most accepted imaging tool for the detection, characterization, and treatment monitoring of ILD [3-7]. Moreover its findings have demonstrated a good correlation with the pulmonary function tests (PFTs) acquiring a prognostic value for ILD $[4,8]$.
Despite these characteristics the correct interpretation of HRCT findings still represents often a problem for the inexperienced physicians since there is a wide interobserver variability even among expert radiologists [9]. Therefore, a quantitative, noninvasive and reliable imaging method able to permit an accurate assessment of ILD in SSc is highly desirable $[10,11]$.

To date, several computerized tools to segment automatically the lung, using HRCT images, have been developed [12]. They include image display (e.g., multiplanar reformations and surface shading for three-dimensional and volume rendering), anatomic image quantitation (e.g., area and volume of airways and lungs), and regional characterization of lung 
tissue (analyzing attenuation, changes in attenuation, and texture patterns in the imaged lung) [12-14]. They also provide computer-derived measures such as mean lung attenuation (MLA) (representing the average global attenuation value of the pulmonary parenchyma), skewness (representing the extent of asymmetry of histograms), and kurtosis (representing the degree of "peakedness" of the histograms) [15]. Additionally, the acquisition of more sophisticated image analysis including the fractal analysis and the adaptive multiple feature method is possible [16].

With respect to the traditional visual interpretation of HRCT lung findings, the automatic computer-based assessment may improve the objectivity, sensitivity, and repeatability of quantitative changes in the lung features. We recently investigated the utility of an open-source Digital Imaging and Communication in Medicine viewer software OsiriX to assess ILD in patients with SSc showing a significant association between the quantitative OsiriX assessment and the conventional HRCT semiquantitative analysis. Results for the reliability of the open-source findings were also acceptable [17].

Taking into account this information we designed the present study aimed to evaluate the performance of a computerized-aided method (CaM) for the quantification of ILD, in patients with SSc and to determine its correlation with respect to both the conventional visual reader-based score (CoVR) and the PFTs findings. The secondary aims were to evaluate the feasibility and interreader reliability of the CaM.

\section{Materials and Methods}

2.1. Patients. Patients with SSc, defined by the American College of Rheumatology (formerly, the American Rheumatism Association) classification criteria [18], were included in the study. SSc patients were classified in limited and diffuse cutaneous involvement ( $1 \mathrm{cSS} c$ and dcSSc, resp.). LcSSc was characterized by thickening of the skin distal to the elbows and knees and proximal to the clavicles (including the face) whereas dcSSc was characterized by thickening of the skin proximal as well as distal to the elbows and knees and including the trunk and the face. Exclusion criteria included absence of recent or current respiratory infection, severe pulmonary hypertension requiring specific treatment, uncontrolled congestive heart failure, known history of asthma, allergic alveolitis, and exposure to organic dusts or clinically significant abnormalities other than interstitial lung disease identified on chest radiography or on HRCT.

2.2. Pulmonary Function Tests. PFTs were performed within 1 week from the lung HRCT assessment by a flow-sensing spirometer and a body plethysmograph connected to a computer for data analysis. PFTs were performed while the patient was at rest in a seated position. These tests consisted of spirometry using a computerised lung analyser (MasterScreen Diffusion, Jaeger GmbH, Höchber, Germany). Forced vital capacity (FVC), forced expiratory volume in $1 \mathrm{~s}$ (FEV1), and the single breath carbon monoxide diffusing capacity of the lung (DLco) were obtained. These parameters of PFT were expressed as percentage of predicted value. At least three measurements were taken for each variable to guarantee repeatability.
2.3. HRCT Assessment and Visual Reader-Based Disease Quantification. All HRCT examinations were performed according to standard protocol using a CT $64 \mathrm{GE}$ light Speed VCT power scanner with a rotation tube scanning time of $0.65 \mathrm{~s}$. Scans were obtained at full inspiration from the apex to the lung base with the patients in the supine position, at $120 \mathrm{kV}$ and $300 \mathrm{mAs}$ and slice thickness and spacing of scans of $1.25 \mathrm{~mm}$ and $7 \mathrm{~mm}$, respectively. HRCT assessment did not include the use of contrast media agents. The parenchymal abnormalities on HRCT were coded and scored in all the images by two independent readers, blinded with respect to the results, according to Warrick et al. [11]. A point value was assigned to each abnormality as follows: ground-glass appearance $=1$; irregular pleural margins $=2$; septal/subpleural lines $=3$; honeycombing $=4$; subpleural cysts $=5$. In each patient the "severity of disease" score was obtained by adding single point values. The mean values of the two independent readers were used as a final control group. An "extent of disease" score was obtained by counting the number of bronchopulmonary segments involved for each abnormality: one to three segments scored as 1; four to nine segments scored as 2; more than nine segments scored as 3 . The severity and extent of disease were then calculated as total HRCT score (range from 0 to 30). The HRCT examinations were randomised and reviewed by two radiologists (E.B and M.C) with more than 15 years of experience in general and thoracic radiology who were unaware of clinical or functional findings. The preliminary agreement between the two radiologists with regard the total HRCT scores was good: intraclass correlation coefficients (ICC) 0.81 .

2.4. Computerized-Aided Scoring Quantification Process. HRCT images were reconstructed and analysed by OsiriX, a DICOM viewer software [19] (OsiriX version 3.9; Apple Computer) on a Mac Mini (2.8 GHz Intel Core 2 Duo Desktop Computer, 16 GB random-access memory; Apple Computer, Cupertino, CA, USA) running Mac Operating System X 10.8.5. After inserting the DVD containing HRCT data in the drive, the DICOM data were automatically extracted from the disc by OsiriX. The DICOM data were stored in the OsiriX using the "Copy linked files to Database folder" under "file" in the OsiriX dropdown menu. The program uses a semiautomated thresholding technique to isolate the lungs from other tissues and structures. For each section, a semiautomatic lung parenchymal segmentation was performed in order to obtain analysis of all images (Figure 1). Then, descriptive parameters of the computer analysis were calculated. The radiodensity of the lung parenchyma isolated from the mediastinum and the thoracic wall ranges between -200 and -1024 . According to Shin et al. [20], the value of radiodensity for ILD was considered from -700 to -500 . So, in the present study, the thresholds of -1024 and -700 were used for the evaluation of the nonfibrotic HRCT lung volume. Adopting these radiodensity values we calculated the pulmonary fibrosis fraction.

Figure 1 illustrates the sequences of the OsiriX segmentation process. A minimal user intervention in the CaM (one author) was required to exclude lung structures not relevant for the assessment (i.e., trachea, blood vessels, and large bronchi near the hilum). 


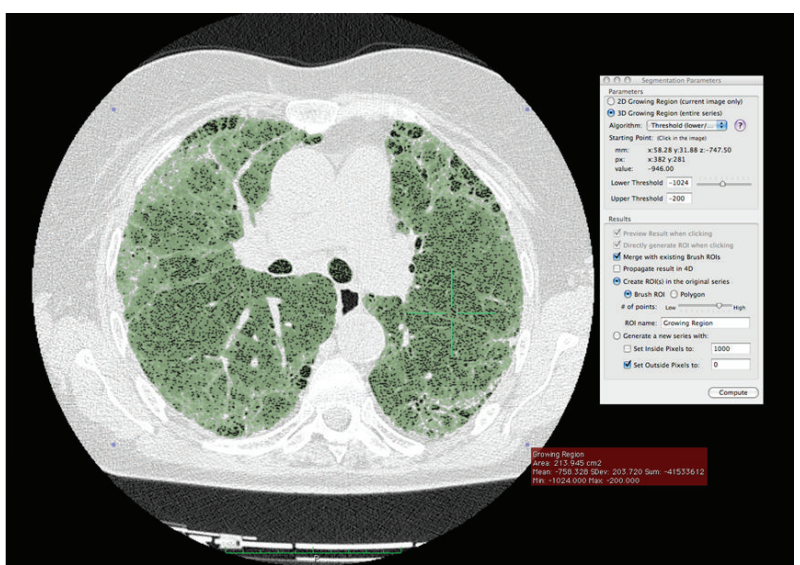

(a)

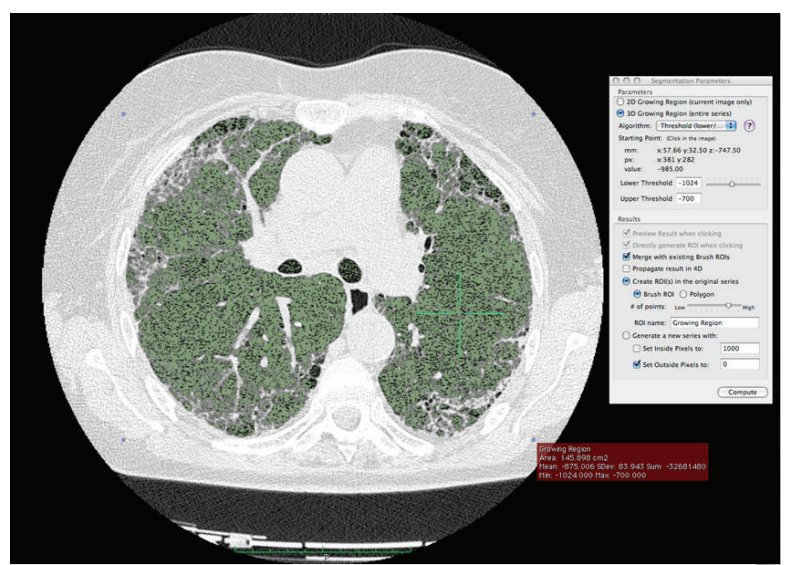

(b)

FIGURE 1: Representative sequences of the OsiriX segmentation process. We have developed the pulmonary fibrosis fraction by the following formula: total HRT lung volume (-1.024 to -200$)$ - nonfibrotic HRCT lung volume $(-1.024$ to -700$)$ divided by total HRT lung volume $(-1.024$ to -200$)$ multiplied by 100 .

2.5. Statistical Analysis. All data were entered into a Microsoft Access database developed for the management of all data. The data were analysed using the SPSS version 11.0 (SPSS Inc., Chicago, IL) and the MedCalc version 10.1 (MedCalc Software, Mariakerke, Belgium). Measurement reproducibility of repeated OsiriX-based assessments and interobserver agreement between the two readers of HRCT were tested using the ICC. This value is an expression of $95 \%$ of all measurements that is expected to be included within the range (limits of agreement). Feasibility of computerized analysis by OsiriX was estimated by comparing the time spent for the quantitative analysis using the CaM with respect to HRCT CoVR semiquantitative analysis by the independent samples " $t$ " test. The relationships among the lung segmentation analysis, the readers, and the PFTs results were calculated using linear regression analysis and Pearson's product moment correlation (" $r$ " values). Student's $t$-test was used to compare two subgroups of the study population for continuous characteristics, and the chi-square test was used for categorical characteristics. Differences corresponding to $P<0.05$ were considered significant.

Receiver operating curve (ROC) analysis was also performed for determination of CaM optimal extent threshold. ROC curve was plotted to determine the area under the curve (AUC) and determine sensitivity, specificity, positive and negative predictive values, and positive likelihood ratio $(\mathrm{LR}+)$. Although there is no official consensus with this regard, fibrotic scores on CoVR, defined according to Warrick et al. [11], were categorised into two groups as follows: $\leq 7$ (mild lung fibrosis) and $>7$ (severe lung fibrosis). A minimum score of 7 on CoVR system would be required to consider HRCT abnormalities in SSc as predictive of pulmonary disease [21]. We used this cut-off as external criteria to dichotomize the patients. The nonparametric Wilcoxon's signed rank test was used for calculation and comparison of the areas under the ROC curves (AUC-ROCs) derived from the sample of patients.

\section{Results}

3.1. Patients. The study group included 79 patients ( 12 male, 67 female, mean age $59 \pm 9.7$ years) with SSc. The average disease duration was $9.3 \pm 5.8$ years. Thirty-eight patients were classified as having $\mathrm{dcSSc}$ (mean age, $61 \pm 9.6$ years; range, 33 to 78 years; disease duration, $10.9 \pm 6.3$ years) and 41 patients were classified as having lcSSc (mean age, $56 \pm 9.2$ years; range, 31 to 69 years; disease duration, $8.5 \pm 5.9$ years). The group of patients having dcSSc, in comparison with lcSSc patients, was older $(62.3 \pm 8.5$ versus $57.1 \pm 9.4$ years; $P<0.05)$ and with a long-term disease $(10.4 \pm 5.4$ versus $8.6 \pm 5.9$ years; $P<0.05)$. The mean $( \pm \mathrm{SD})$ time interval between PFTs and HRCT was $4.5 \pm 1.5$ days (range: $0-7$ days). On PFTs, average FVC was $89.6 \pm 9.6 \%$ of predicted, average FEV1 was $83.8 \pm$ $8.7 \%$ of predicted, and average DLco was $70.1 \pm 15.9 \%$ of predicted. FEV1 and DLco were statistically different in the two groups of SSc patients $(P<0.001)$. All patients displayed HRCT findings of ILD (detected by the readers: average total HRCT score $=12.1 \pm 6.9$ ). Similarly, the percentage of extent of lung diseases measured by CaM was significantly higher, independently of the gender, in patients with dcSSc $(22.1 \pm$ $9.7 \%$ versus $16.3 \pm 9.0 ; P=0.008)$.

\subsection{Correlation between Computerized-Aided Method Results,} Visual Reader-Based Scoring Method, and PFTs. A close correlation between CaM results and CoVR was observed $(r=0.829 ; P<0.0001)$ (Figure 2). The computerizedaided scores showed a moderate to highly significant negative correlation with forced vital capacity (FVC) $(r=-0.490 ; P<$ $0.0001)$, forced expiratory volume in $1 \mathrm{~s}$ (FEV1) $(r=-0.675$; $P<0.0001)$ and the single breath carbon monoxide diffusing capacity of the lung (DLco) $(r=-0.653 ; P<0.0001)$ (Figure 3).

\subsection{Determination of a CaM Optimal Extent Threshold.} Figure 4 shows the ROC curve representation of CaM values for threshold of HRCT findings. The ROC analysis demonstrated excellent performance with an AUC of 0.886 


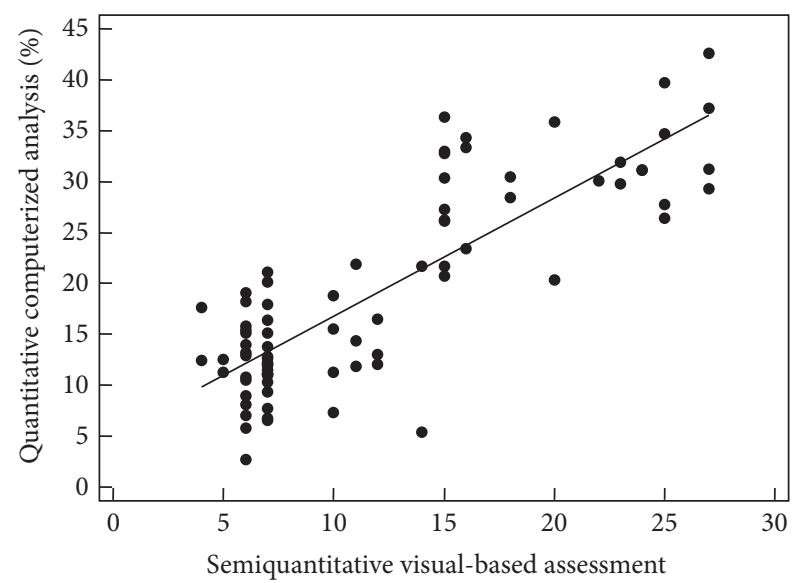

FIGURE 2: Scatter plots with regression line, illustrating the correlation between computerized-based analysis and visual reader-based scoring method.

(SE, 0.041) (Figure 4). For the CaM, a cut-off $20 \%$ of lung involvement represented the best compromise between sensitivity (75.6\%) and specificity (97.4\%), with a positive predictive value of 92.2 and a $+\mathrm{LR}$ of 29.9 . A value of $10 \%$ for quantitative lung disease increased the sensitivity to $95.1 \%$ but decreased the specificity to $30.7 \%$, whereas a value of $30 \%$ increased the specificity to $100 \%$ but decreased the sensitivity to $34.1 \%$ (Table 1 ).

3.4. Feasibility. The mean time spent completing the quantitative evaluation by CaM was $1.3 \mathrm{~min}$ (range 1 to $2.1 \mathrm{~min}$ ) whereas it was $10.9 \mathrm{~min}$ (range 5.9 to $14.9 \mathrm{~min}$ ) adopting the semiquantitative visual assessment. The difference was highly significant (Student's $t$-test, $P<0.0001$ ).

\section{Discussion}

An accurate characterization and quantification of ILD is essential for a correct clinical management of patients with SSc $[10,11,22]$. Our results indicate that the CaM analysed by OsiriX provides a good concurrent validity, reliability, and feasibility for the assessment of ILD in patients with SSc. Considering the promising advent of user friendly software's [19], this approach may be effectively used in both clinical practice and research setting.

To date different visual-based semiquantitative approaches to assess ILD have been proposed $[11,22]$. In most of them the final score was calculated either by agreement between two reviewers or by obtaining the mean of the reading scores by two reviewers. Warrick et al. [11] have proposed a semiquantitative method in patients with SSc which allows the evaluation of the different patterns of abnormalities, rated according to the severity and extent of lung damage, through a total overall HRCT score. Kazarooni et al. [22] have divided each lung into three zones (lung apex to aortic arch, aortic arch to inferior pulmonary veins, and inferior pulmonary veins to lung bases) and scored the extent of lung abnormality of each zone on a scale ranging from 0 to 4 . More recently, a simplified scoring system based on the grade of the lung involvement more or less than $25 \%$ has also been suggested [4].

The CoVR method plays an important role in the interpretation of ILD patterns. Moore et al. [23] have shown that a simple and quick grading system for the extent of total lung disease on HRCT has prognostic significance in SSc, even after adjustment for other prognostic covariates. Similarly, Goh et al. [4] have demonstrated that an easily applicable limited/extensive staging system for SSc-ILD, based on combined evaluation with HRCT and PFTs, provides discriminatory prognostic information. In particular, the risk of death in SSc-ILD and, separately, progression of disease rose strikingly when the overall percentage of lung involved on HRCT exceeded 20\% [4]. This threshold value was defined using formal CoVR scoring systems, which is seldom practicable in routine practice.

Although the CoVR is currently the most popular method used [24], it has several disadvantages such as subjectivity and difficulty in estimating accurately the different components of disease (honeycombing, reticular, and linear ground-glass opacity). A further difficulty is represented by the complex task of integrating the extent of the abnormalities seen on several HRCT slices and deriving a quantitative measure of the total extent of abnormality of a lung zone or within the lung. Finally, CoVR scoring systems provide lack of reproducibility, with larger interreader and intrareader variation [9]. Compared with visual-based assessments, CaM scores are demonstrated to improve objectivity, sensitivity, and repeatability when measuring the quantitative changes in ILD $[16,17]$.

As mentioned before, our preliminary experience using this system in SSc patients showed a high agreement with respect to the semiquantitative HRCT analysis performed by experienced radiologists and a significant association between the descriptive parameters by both the quantitative OsiriX assessment and the HRCT semiquantitative analysis [17]. It has been previously shown that there is a significant variability in the lung density in normal individuals, and this factor should be taken into account when considering the use of CT lung density mapping for the assessment of pulmonary disease. However, the radiodensity of the lung parenchyma isolated from the mediastinum and the thoracic wall ranges between -200 and -1024 . CT attenuation of normal lung parenchyma is reported to range from -800 to $-900 \mathrm{HU}$, depending on inspiration or expiration, on the level of inspiration achieved for the scan, and on anatomical location that is ventral or dorsal portion [25]. Shin et al. [20] defined the area with attenuation between -500 and -700 as the value of radiodensity for ILD. The author included both ground-glass opacity and reticular opacity. Contrary Yabuuchi et al. [26] used the thresholds of -500 and $-800 \mathrm{HU}$ for the evaluation of ground-glass opacity. Moreover the CT attenuation values for consolidation and ground-glass opacity were separated and the radiodensity of $-500 \mathrm{UH}$ was selected as the thresholds between consolidation and groundglass opacity. However, the application of a threshold value of $-800 \mathrm{HU}$ may include small peripheral pulmonary vessels and cause an overestimation of interstitial lung disease [27]. In our method, in agreement with Shin et al. [20], -700 HU is selected as the predefined threshold to obtain lung regions. 


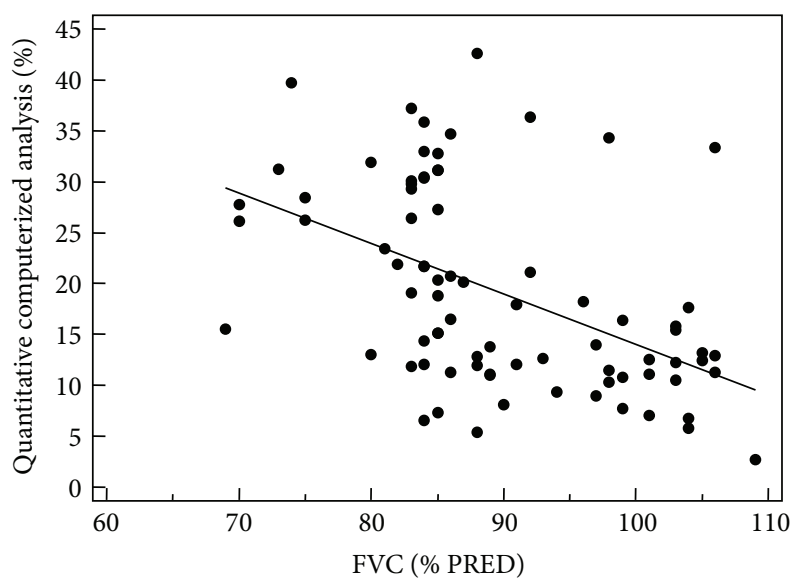

(a)

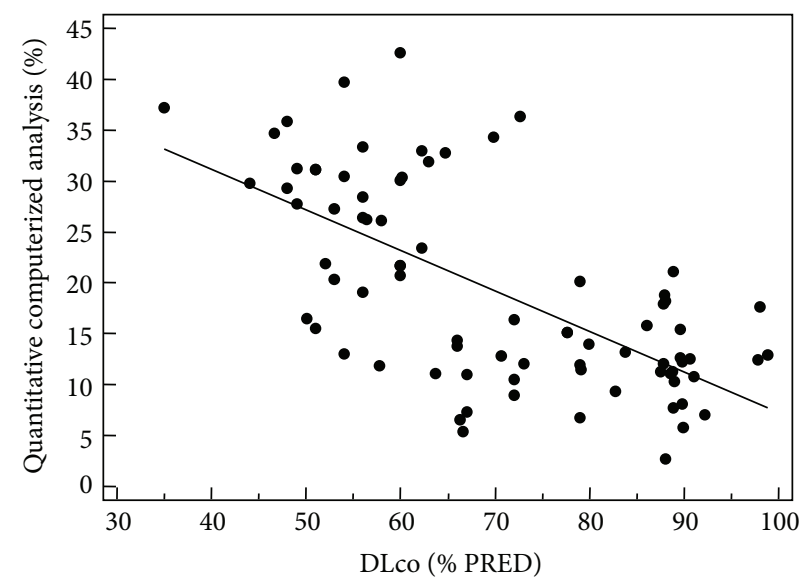

(b)

FIGURE 3: Scatter plots showing correlations between PFTs and quantitative computerized analysis of pulmonary fraction with regression line. Each circle shows a single patient data.

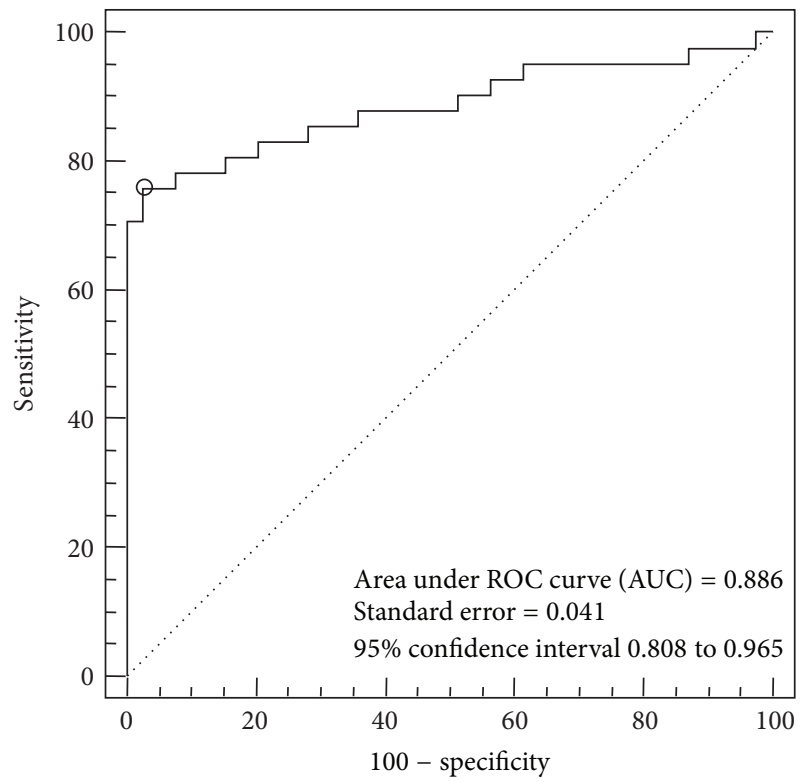

FIGURE 4: ROC curve for determination of CaM optimal extent threshold. The circle on the curve shows optimal cut-off point, corresponding with the maximum sum of sensitivity and specificity.

Our current results confirmed that the quantitative OsiriX assessment system correlate well with visual-based scoring techniques for the detection of HRCT extent and severity of disease [17]. The percentage of extent of lung disease showed a significant correlation $(P<0.0001)$ with FVC, $\mathrm{FEV}_{1}$, and DLco.

In a purely clinical context, we have shown that CaM scoring system of ILD may have several advantages in the management of SSc patients. First, the advantage of the OsiriXbased measurement is the use of a continuous scale, rather than a categorical Likert scale. The continuous computerized scoring, in comparison with categorical rating used in the visual (semiquantification) assessment, provides greater power for detecting a treatment effect within a given sample size or allows an approximately $50 \%$ reduction of the sample size [28]. The possibility of having the percentage extent of total lung disease, easily obtainable from the rheumatologist in a clinical outpatient setting with a simple and rapid procedure, represents a clear advantage for the assessment of responsiveness including prognostic value data. Secondly, the OsiriX segmentation algorithm proved to be time-efficient, reproducible, and requiring less than two minutes for the total lung evaluation. A third advantage is that OsiriX-based computerized scoring system can be implemented in the setting of a multicenter trial in SSc-ILD using digitized HRCT images. Finally, OsiriX is user friendly open-source software $[17,19]$ that even rheumatologists can easily manipulate and generate $3 \mathrm{D}$ reconstructed images and acquire whole images of $3 \mathrm{D}$ anatomical structures. The training in using OsiriX software can be easily and quickly completed [19], providing clinicians with a valuable tool for the evaluation of disease extent and interpretation of patterns of pulmonary function impairment in SSc patients.

We are aware of some limitations in our study. First, the diagnosis of pulmonary fibrosis was based on radiological findings, not by histological examination. Secondly, the CaM scoring system used in this study focuses on quantification of total disease extent and lacks a differentiation between different radiographic patterns, but the clinical significance of these HRCT features is as yet unknown. However, the disease extent has been shown to be a strong predictor of functional pulmonary impairment. Furthermore, our quantitative evaluation did not focus on anatomic compartments of the lung; however, in comparison to emphysema, ILD tends to be widespread. Formal HRCT scoring, especially in clinical trials, is commonly performed using predefined anatomic levels rather than pulmonary lobes, as HRCT examinations are still widely performed due to radiation protection. Third, the use of our density mask method for the quantitative analysis of ILD could not discriminate accurately 


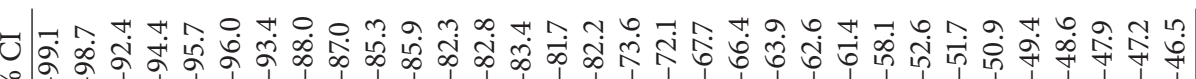

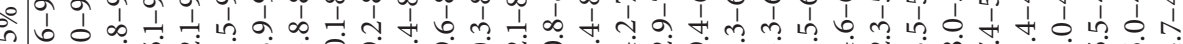

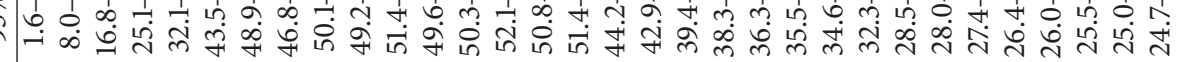

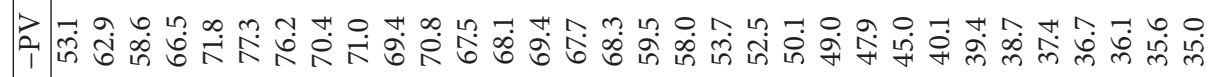

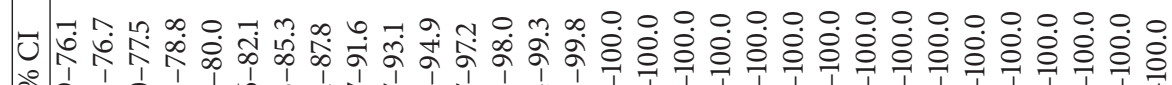

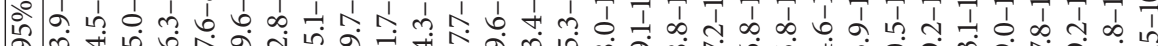

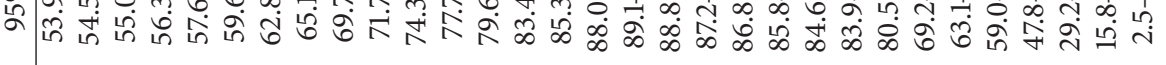

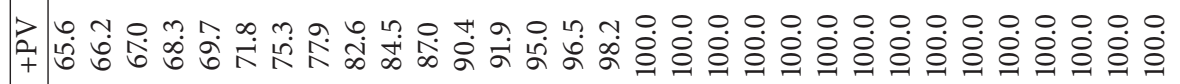

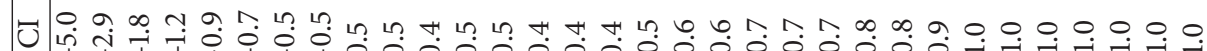

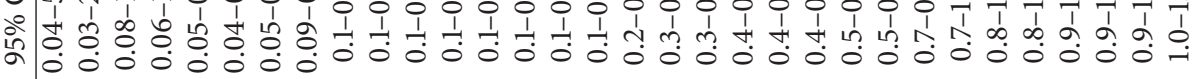

ชั 童

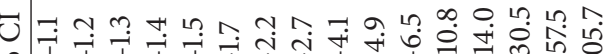

h人 ô.

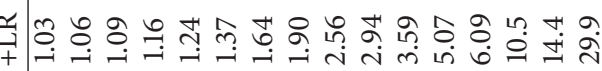

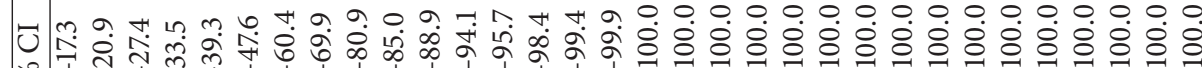

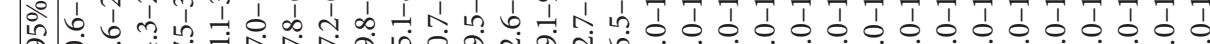

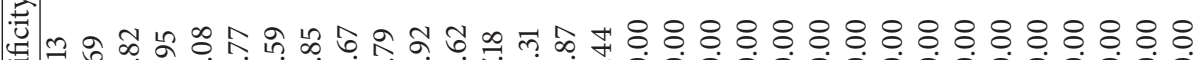
के में

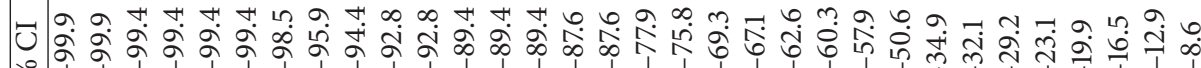

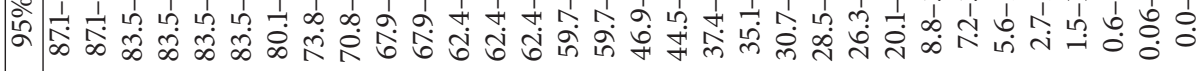

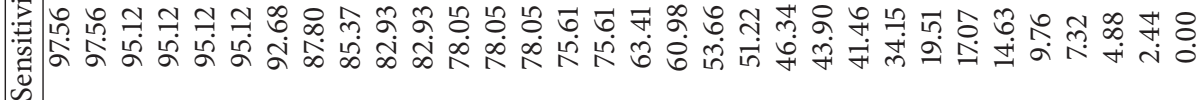

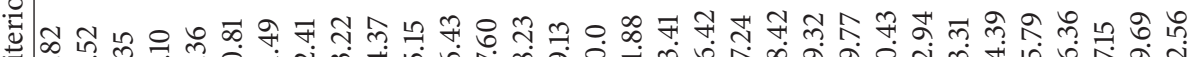

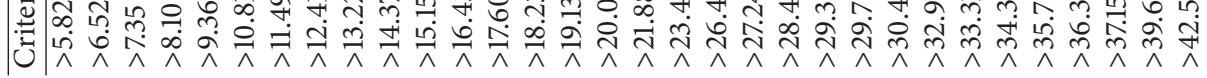


the low attenuation areas of honeycombing from the normal lung density when honeycombing cysts are present which may underestimate the ILD severity. Therefore, the discrepancy of quantification between the CaM scoring systems and CoVR method may be intrinsic to the densitometric analysis. Regarding this intrinsic discrepancy, the usefulness of the automated system was criticized due to the usual presence of lung increased density (ground-glass opacities) and decreased density (cystic spaces, honeycombing) [29]. Finally, the sample size of our study was limited and the effect of pulmonary hypertension was not assessed. Therefore there might be a limit in the comparison with measures of disease severity.

In conclusion, our results showed that the CaM using an open-source software DICOM application-OsiriX-may assist the rheumatologist analysis of lung HRCT data and provides an objective method for supplementing subjective visual-based grading of the extent of ILD to achieve precise and reader-independent quantification. Compared with previous in-house software, OsiriX will enable wider use, resulting in easier computer-aided technique application in routine practice and better communication among different hospitals.

Computer-derived extent of total lung disease appears as discriminant method and, therefore, can help to produce an objective measure and to obtain prognostic information in SSc-ILD. Although these encouraging data require further validation in prospective studies, we believe that the CaM may improve the ability of rheumatologists to quantify accurately the extent of ILD in SSc patients in both daily clinical practice and clinical trials.

\section{Disclosure}

All the authors declare that they have not received any financial support or other benefits from commercial sources for the work reported in this paper nor any other financial interests that could create a potential conflict of interest or the appearance of a conflict of interests with regard to the work.

\section{Conflict of Interests}

The authors would like to make the following statements with regard to their conflict of interests/financial disclosures. Fausto Salaffi has attended advisory board meetings for Bristol-Myers Squibb, Abbott Immunology, Wyeth Lederle, and Pfizer and has received research support from BristolMyers Squibb. Marina Carotti has attended advisory board meetings for Abbott Immunology, Wyeth. Alessandro Ciapetti has attended advisory board meetings and has obtained speaking fees for Bristol-Myers Squibb. Marwin Gutierrez has attended advisory board meetings, scientific consultancies, and has obtained speaking fees for Abbott Immunology, AbbVie, UCB Pharma, Esaote S.p.A, Bristol-Myers Squibb, Merck Sharp \& Dohme. Gianfranco Ferraccioli, Elisabetta Bichisecchi, Gianmarco Giuseppetti, and Silvia Bosello declare no financial conflict of interests.

\section{Authors' Contribution}

Fausto Salaffi participated in the design of the study and the acquisition and interpretation of data, performed the statistical analysis and the drafting of the paper, and gave final approval of the version of the paper to be published. Marina Carotti and Elisabetta Bichisecchi performed the HRCT examinations and their relative interpretation and were involved in revising the paper for important intellectual content. Alessandro Ciapetti, Marwin Gutierrez, Gianmarco Giuseppetti, and Silvia Bosello made substantial contributions to the conception and design of the study, participated in the acquisition of data, and were involved in revising the paper for important intellectual content. Gianfranco Ferraccioli participated in the conception of the study and data interpretation, was involved in drafting the paper, and gave final approval of the version of the paper to be published. All authors read and approved the final paper.

\section{References}

[1] C. Ferri, G. Valentini, F. Cozzi et al., "Systemic sclerosis: demographic, clinical, and serologic features and survival in 1,012 Italian patients," Medicine, vol. 81, no. 2, pp. 139-153, 2002.

[2] A. U. Wells, M. B. Rubens, R. M. Du Bois, and D. M. Hansell, "Serial CT in fibrosing alveolitis: prognostic significance of the initial pattern," The American Journal of Roentgenology, vol. 161, no. 6, pp. 1159-1165, 1993.

[3] C. P. Simeon, L. Armadans, V. Fonollosa et al., "Mortality and prognostic factors in Spanish patients with systemic sclerosis," Rheumatology, vol. 42, no. 1, pp. 71-75, 2003.

[4] N. S. L. Goh, S. R. Desai, S. Veeraraghavan et al., "Interstitial lung disease in systemic sclerosis: a simple staging system," American Journal of Respiratory and Critical Care Medicine, vol. 177, no. 11, pp. 1248-1254, 2008.

[5] M. Remy-Jardin, J. Remy, B. Wallaert, D. Bataille, and P.Y. Hatron, "Pulmonary involvement in progressive systemic sclerosis: sequential evaluation with $\mathrm{CT}$, pulmonary function tests, and bronchoalveolar lavage," Radiology, vol. 188, no. 2, pp. 499-506, 1993.

[6] J. G. Goldin, D. A. Lynch, D. C. Strollo et al., "High resolution CT findings in scleroderma-related lung diseases: findings from Scleroderma lung study," Chest, vol. 134, pp. 358-367, 2008.

[7] R. Giacomelli, G. Valentini, F. Salsano et al., "Cyclophosphamide pulse regimen in the treatment of alveolitis in systemic sclerosis," Journal of Rheumatology, vol. 29, no. 4, pp. 731-736, 2002.

[8] A. Tochimoto, Y. Kawaguchi, M. Hara et al., "Efficacy and safety of intravenous cyclophosphamide pulse therapy with oral prednisolone in the treatment of interstitial lung disease with systemic sclerosis: 4-year follow-up," Modern Rheumatology, vol. 21, no. 3, pp. 296-301, 2011.

[9] D. Khanna, C.-H. Tseng, N. Farmani et al., "Clinical course of lung physiology in patients with scleroderma and interstitial lung disease: analysis of the Scleroderma Lung Study Placebo Group," Arthritis and Rheumatism, vol. 63, no. 10, pp. 30783085, 2011.

[10] J. G. Goldin, "Quantitative CT of the lung," Radiologic Clinics of North America, vol. 40, no. 1, pp. 145-162, 2002.

[11] J. H. Warrick, M. Bhalla, S. I. Schabel, and R. M. Silver, "High resolution computed tomography in early scleroderma lung 
disease," Journal of Rheumatology, vol. 18, no. 10, pp. 1520-1528, 1991.

[12] G. Camiciottoli, I. Orlandi, M. Bartolucci et al., "Lung CT densitometry in systemic sclerosis: correlation with lung function, exercise testing, and quality of life," Chest, vol. 131, no. 3, pp. 672681, 2007.

[13] J. K. Leader, B. Zheng, R. M. Rogers et al., "Automated lung Segmentation in X-ray computed tomography: development and evaluation of a heuristic threshold-based scheme," Academic Radiology, vol. 10, no. 11, pp. 1224-1236, 2003.

[14] J. Wang, F. Li, and Q. Li, "Automated segmentation of lungs with severe interstitial lung disease in CT," Medical Physics, vol. 36, no. 10, pp. 4592-4599, 2009.

[15] P. G. Hartley, J. R. Galvin, G. W. Hunninghake et al., "Highresolution CT-derived measures of lung density are valid indexes of interstitial lung disease," Journal of Applied Physiology, vol. 76, no. 1, pp. 271-277, 1994.

[16] R. Uppaluri, E. A. Hoffman, M. Sonka, G. W. Hunninghake, and G. McLennan, "Interstitial lung disease: a quantitative study using the adaptive multiple feature method," American Journal of Respiratory and Critical Care Medicine, vol. 159, no. 2, pp. 519525, 1999.

[17] A. Ariani, M. Carotti, M. Gutierrez et al., "Utility of an opensource DICOM viewer software (OsiriX) to assess pulmonary fibrosis in systemic sclerosis: preliminary results," Rheumatology International, vol. 34, no. 4, pp. 511-516, 2014.

[18] Subcommittee for Scleroderma Criteria of the American Rheumatism Association Diagnostic and Therapeutic Criteria Committee, "Preliminary criteria for the classification of systemic sclerosis (scleroderma)," Arthritis \& Rheumatology, vol. 23, pp. 581-590, 1980.

[19] A. Rosset, L. Spadola, and O. Ratib, "OsiriX: an open-source software for navigating in multidimensional DICOM images," Journal of Digital Imaging, vol. 17, no. 3, pp. 205-216, 2004.

[20] K. E. Shin, M. J. Chung, M. P. Jung, B. K. Choe, and K. S. Lee, "Quantitative computed tomographic indexes in diffuse interstitial lung disease: correlation with physiologic tests and computed tomography visual scores," Journal of Computer Assisted Tomography, vol. 35, no. 2, pp. 266-271, 2011.

[21] E. Diot, E. Boissinot, E. Asquier et al., "Relationship between abnormalities on high-resolution CT and pulmonary function in systemic sclerosis," Chest, vol. 114, no. 6, pp. 1623-1629, 1998.

[22] E. A. Kazarooni, F. J. Martinez, A. Flint et al., “Thin-section CT obtained at $10-\mathrm{mm}$ increments versus limited three-level thin-section CT for idiopathic pulmonary fibrosis: correlation with pathologic scoring," American Journal of Roentgenology, vol. 169, no. 4, pp. 977-983, 1997.

[23] O. A. Moore, N. Goh, T. Corte et al., "Extent of disease on high-resolution computed tomography lung is a predictor of decline and mortality in systemic sclerosis-related interstitial lung disease," Rheumatology, vol. 52, no. 1, pp. 155-160, 2013.

[24] R. K. Rienmuller, J. Behr, W. A. Kalender et al., "Standardized quantitative high resolution CT in lung diseases," Journal of Computer Assisted Tomography, vol. 15, no. 5, pp. 742-749, 1991.

[25] L. J. Rosenblum, R. A. Mauceri, D. E. Wellenstein et al., "Density patterns in the normal lung as determined by computed tomography," Radiology, vol. 137, no. 2, pp. 409-416, 1980.

[26] H. Yabuuchi, Y. Matsuo, H. Tsukamoto et al., "Evaluation of the extent of ground-glass opacity on high-resolution CT in patients with interstitial pneumonia associated with systemic sclerosis: comparison between quantitative and qualitative analysis," Clinical Radiology, vol. 69, no. 7, pp. 758-764, 2014.
[27] K. Marten, V. Dicken, C. Kneitz et al., "Computer-assisted quantification of interstitial lung disease associated with rheumatoid arthritis: preliminary technical validation," European Journal of Radiology, vol. 72, no. 2, pp. 278-283, 2009.

[28] H. J. Kim, M. S. Brown, R. Elashoff et al., "Quantitative texturebased assessment of one-year changes in fibrotic reticular patterns on HRCT in scleroderma lung disease treated with oral cyclophosphamide," European Radiology, vol. 21, no. 12, pp. 2455-2465, 2011.

[29] A. U. Wells, D. M. Hansell, M. B. Rubens et al., "Fibrosing alveolitis in systemic sclerosis: indices of lung function in relation to extent of disease on computed tomography," Arthritis and Rheumatism, vol. 40, no. 7, pp. 1229-1236, 1997. 


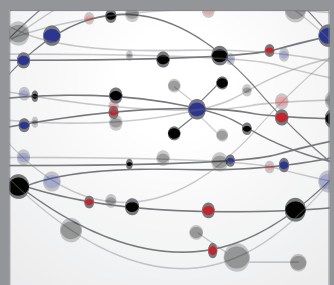

The Scientific World Journal
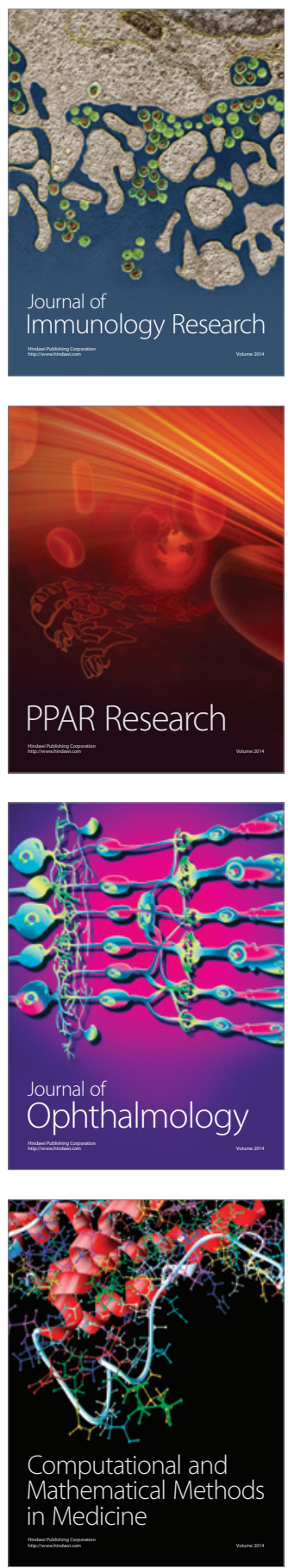

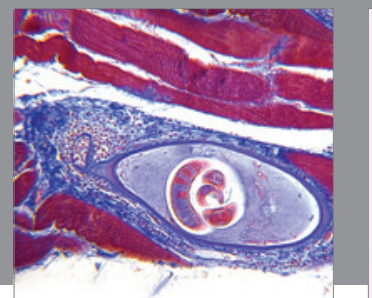

Gastroenterology

Research and Practice
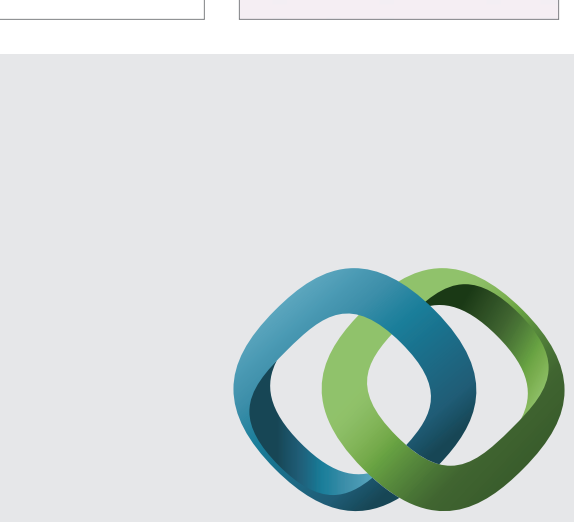

\section{Hindawi}

Submit your manuscripts at

http://www.hindawi.com
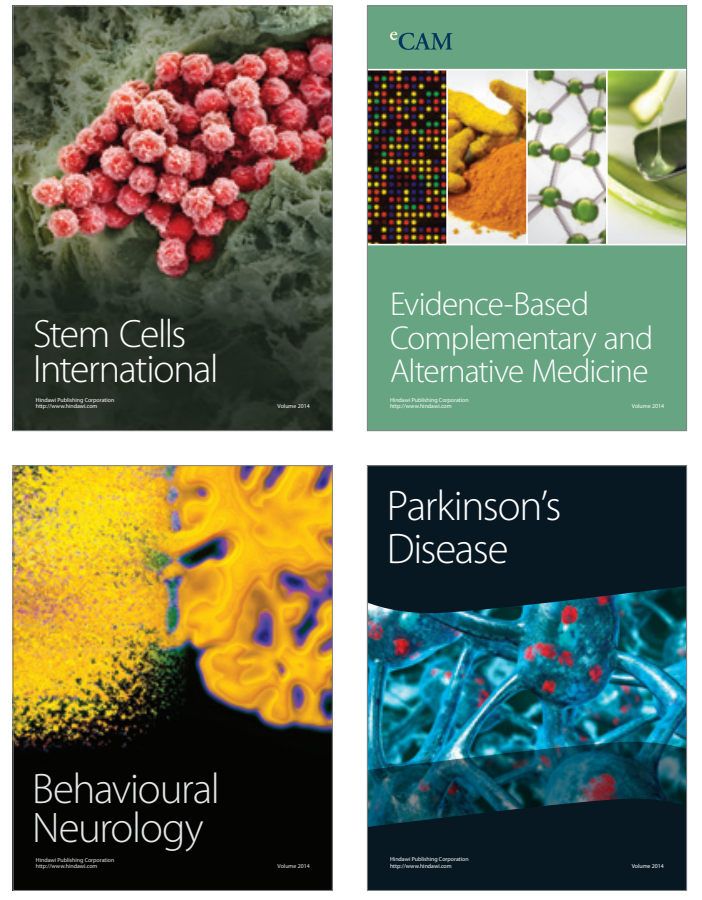
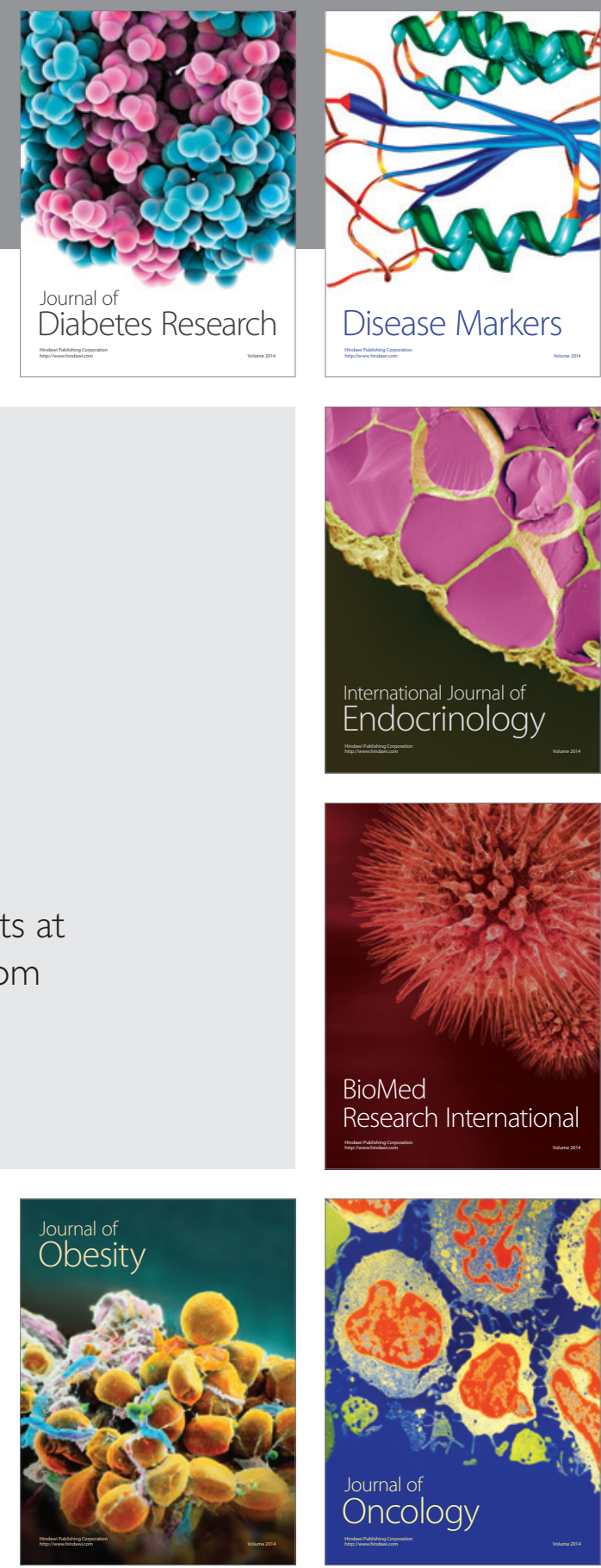

Disease Markers
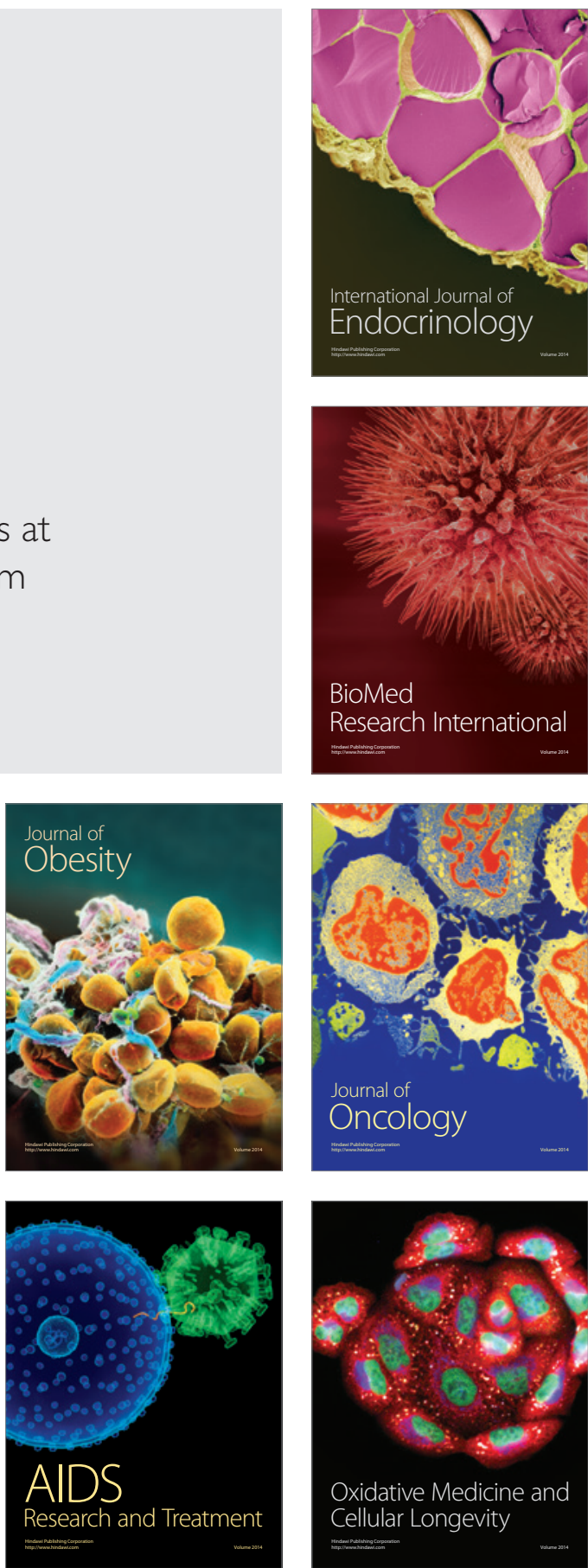